\title{
Photodegradation of pesticides and application of bioanalytical methods for their detection*
}

\author{
Mladen Franko ${ }^{1, \ddagger}$, Mohamed Sarakha ${ }^{2}$, Anja Čibej ${ }^{1}, \#$, Aleš Boškin ${ }^{1}$, \\ Mojca Bavcon ${ }^{1}$, and Polonca Trebše ${ }^{1}$ \\ ${ }^{1}$ School of Environmental Sciences, Nova Gorica Polytechnic, Vipavska 13, \\ P.O.B. 301, SI-5001, Nova Gorica, Slovenia; ${ }^{2}$ Laboratoire de Photochimie \\ Moleculaire et Macromoleculaire, UMR Université Blaise Pascal, CNRS 6505, \\ Aubiere, France
}

\begin{abstract}
Photodegradation of coumaphos and azinphos-methyl in the air, in oxygen, and in nitrogen atmosphere (quartz reactor equipped with six 18-W lamps with the maximum emission intensity at $310 \mathrm{~nm}$ ) was studied. The fastest reaction occurred with coumaphos in nitrogen atmosphere (complete decomposition in $10 \mathrm{~min}$ ), where the formation of a new compound was also detected by high-performance liquid chromatography (HPLC). In the case of azinphos-methyl, no additional signals in HPLC chromatogram were observed. The rearrangement mechanism, involving a triplet-state activation complex and the formation of an acetylcholinesterase (AChE)-inhibiting oxo analog of coumaphos was verified and confirmed by laser flash photolysis.

The suitability of bioanalytical flow-injection analysis (FIA) systems, based on AChE inhibition and spectrophotometric or thermal lens spectrometric detection for rapid and sensitive screening in food quality control was demonstrated. Owing to the high sensitivity of thermal lens spectrometry (TLS), several steps in sample preparation can be avoided (preconcentration, purification, isolation) and incubation times reduced. High sample throughputs $\left(10 \mathrm{~h}^{-1}\right)$ are, however, also achievable for medium toxic oxo organophosphorus (OP) pesticides using spectrophotometric detection providing limits of detection (LODs) at the level of $10 \mathrm{ppb}$ malaoxon equivalents, which is still about 50 times below maximal residue levels. Testing of the method for practical application on a set of 60 samples gave no false negative results and a level of $1.7 \%$ false positives.
\end{abstract}

Keywords: Pesticides; photodegradation; bioanalytical methods; flow-injection analysis; acetylcholinesterase.

\section{INTRODUCTION}

Owing to extensive use in agriculture, organophosphorus (OP) pesticides are among the most widely spread pollutants in the environment. They inhibit acetylcholinesterase (AChE) activity not only in insects, but can also affect the nervous system of other organisms as well as humans [1,2]. Investigation and understanding of the stability and transformations of OP compounds in the environment has, therefore, been the subject of extensive studies, since it is important from the toxicological aspect as well as

\footnotetext{
*Paper based on a presentation at the $4^{\text {th }}$ International Conference of the Chemical Societies of the South-Eastern European Countries (ICOSECS-4), Belgrade, Serbia and Montenegro, 18-21 July 2004. Other presentations are published in this issue, pp. $1655-1752$.

¥Corresponding author

${ }^{\#}$ Current address: Ministry of Defence, Kardeljeva ploščad 25, 1000 Ljubljana, Slovenia.
} 
for the search and development of efficient methods for detoxification and removal of OP compounds as hazardous wastes. Simultaneously, new analytical methods have been developed for detection of OP compounds and resulting degradation products in various samples, and for studies of their toxicities.

OP esters are very susceptible to hydrolysis, which is, therefore, their most common degradation pathway [2]. Hydrolysis can occur when dissolved metal ions enhance the rate of hydrolysis by catalysis [3] or by the $\mathrm{pH}$ [4]. Dissolved matter and temperature also affect the hydrolysis of OP compounds $[5,6]$. In fact, it was claimed that the presence of humic substances, metal oxides, and the soil structure could affect the decomposition rate [5-7].

Light causes photolytic degradation of various OP compounds [8-11]. Degradation can occur by direct and indirect photolysis. In direct photolysis, the pesticide absorbs UV light and afterwards reacts with substances in the environment or decomposes by itself. However, indirect photolysis is more common. This is caused by oxygen and hydroxy or peroxy radicals, which are produced by photolysis in humic or inorganic compounds [3]. Some studies included simulation of the influence of water disinfection processes such as chlorination [3] and ozonization [12,13] on the transformation of diazinon into its metabolites. Photochemical reactions are one of the most often transformations of pesticides in the environment. Therefore, investigations of photodegradation processes can provide better knowledge on transformations and degradation processes of OP pesticides in the environment and their oxidation/degradation rate.

An essential step of detoxification processes should also be the determination of metabolite's toxicity, which requires a simple, rapid, and inexpensive screening method. Most of the conventional methods for determination of organophosphates, such as high-performance liquid chromatography (HPLC), gas chromatography (GC), and gas chromatography/mass spectrometry (GC/MS), are labor-intensive, time-consuming, and require expensive instrumentation. Thus, bioanalytical methods such as biosensors were recently developed and found useful as an alternative method for screening and toxicity tests. Among the bioanalytical methods available for detection of organophosphate pesticides, enzymatic methods based on cholinesterase (ChE) inhibition were widely investigated and a detailed review on this subject has been published [14]. By applying these devices, it is possible to reliably identify potentially hazardous samples, which need further analysis in laboratory using more expensive and timeconsuming analytical approaches. Unfortunately, most of the biosensors lack sensitivity sufficient to determine concentrations of pesticides present in environmental samples and/or require long incubation times. A new approach utilizes photothermal detection based on thermal lens spectrometry (TLS) in combination with a bioanalytical flow-injection analysis (FIA) system [15] to achieve low limits of detection and relatively short time of analysis [16-18].

Since the mechanism of photodegradation determines also the toxicity of metabolites formed, it is our objective to study photodegradation processes of different organophosphates in combination with toxicity measurements using bioanalytical methods, which offer the information about the overall anti$\mathrm{ChE}$ toxicity of samples. The development of photothermal bioanalytical FIA methods described in this contribution aims for their application as a screening test and an early warning system for contamination with organophosphate pesticides.

\section{EXPERIMENTAL}

\section{Chemicals}

Coumaphos [O,O-diethyl $O$-(3-chloro-4-methyl-7-coumarinyl)phosphothioate, $99.5 \%]$ and azinphos-methyl $\{O, O$-dimethyl $S$-[(4-oxo-1,2,3-benzotriazin-3(4H)-yl)methyl]phosphodithioate, $98.6 \%$, diazinon $(O, O$-diethyl $O$-2-isopropyl-6-methylpyrimidin-4-yl phosphothioate, $99.8 \%)$, malathion [S-1,2-bis(ethoxycarbonyl)ethyl $O, O$-dimethyl phosphodithioate, $99.5 \%$ ], fenitrothion $(O, O$-dimethyl $O$-4-nitro- $m$-tolyl phosphothioate, $99 \%$ ), malaoxon [ $O, O$-dimethyl $S$-1,2-bis(ethoxycarbonyl)ethyl phosphothioate, $99 \%$ ] were purchased from Pestanal, Riedel de Haen. 
Tetrabutylammonium hydrogensulfate ( $\geq 97 \%), N$-bromosuccinimide $(79 \%)$, and DTNB [5,5'-dithiobis (2-nitrobenzoic acid)] were purchased from Fluka; ascorbic acid (99.7\% from Kemika), hexane $(99 \%)$, and sodium sulfate $\mathrm{Na}_{2} \mathrm{SO}_{4}(99 \%)$ from Riedel de Haen; ethylacetate $(99.8 \%$ ), acetonitrile (HPLC grade), and nitric acid $\mathrm{HNO}_{3}(65 \%)$ ) from Carlo Erba; glutaraldehyde (25\% aqueous solution) from Merck, acetylthiocholine iodide, 3-aminopropylethoxysilane, and AChE, type III (from electric Eel—aqueous solution 1100 units $/ \mathrm{mg}$ protein or $1.2 \mathrm{mg}$ protein $/ \mathrm{mL}$ ); controlled-pore glass (CPG), surface area $94 \mathrm{~m}^{2} / \mathrm{g}$ from Sigma, and extraction cartridges C18-E $(1 \mathrm{~mL} / 100 \mathrm{mg})$ from Phenomenex.

\section{Analysis of pesticides}

Determination of coumaphos and azinphos-methyl was performed by HPLC on a Zorbax C8 column $(4.6 \mathrm{~mm}$ i.d. $\times 25 \mathrm{~cm}$ ). The mobile phase pumped at $1 \mathrm{~mL} / \mathrm{min}$ flow rate used in the case of coumaphos was a $30: 70$ mixture of $0.01 \mathrm{M}$ tetrabutylammonium hydrogensulfate $(3.3954 \mathrm{~g} / \mathrm{L}$ of deionized water) and acetonitrile, while a 40:60 mixture at the same flow rate was used for azinphos-methyl. The chromatograms were recorded by measuring the absorbance at $313 \mathrm{~nm}$. Coumaphos was eluted at $9.1 \mathrm{~min}$ and azinphos-methyl at $7.54 \mathrm{~min}$.

Determination of parent pesticides, diazinon, malathion, and fenitrothion and their oxidation products, diazoxon, malaoxon, and fenitrotion-oxon, was performed by GC/MS analysis using gas chromatograph Varian 3900 coupled with mass detector Varian Saturn 2100 T and equipped with Varian column-fused silica CP-Sil 8 low bleed. Chromatographic conditions were as follows: injector temperature $220^{\circ} \mathrm{C}$, split ratio $20: 1$, initial temperature on the column $80{ }^{\circ} \mathrm{C}$, final temperature $290{ }^{\circ} \mathrm{C}$ with the increase rate $10^{\circ} \mathrm{C} / \mathrm{min}$.

\section{Photodegradation experiments}

For the degradation experiments, the amount of $4.1 \mathrm{mg}$ of coumaphos was dissolved in $1 \mathrm{~L}$ of the mixture of deionized water and acetonitrile (80:20) or $2.0 \mathrm{mg}$ of azinphos-methyl was dissolved in $0.2 \mathrm{~L}$ of deionized water. The experiments were performed in a $200-\mathrm{mL}$ photoreactor equipped with six $18-\mathrm{W}$ lamps with the maximum emission intensity at $310 \mathrm{~nm}$.

\section{Oxidation procedure for toxicity tests}

For bioanalytical detection of thiono OP compounds, $0.5 \mathrm{~mL}$ of $N$-bromosuccinimide (NBS, $0.4 \mathrm{~g} / \mathrm{L}$ ) was added to $50 \mathrm{~mL}$ of samples, containing OP pesticides. The mixture was stirred for $5 \mathrm{~min}$ at room temperature, and $0.5 \mathrm{~mL}$ of ascorbic acid $(4 \mathrm{~g} / \mathrm{L})$ was added. Stirring continued at room temperature for another $5 \mathrm{~min}$. Part of the sample was used for toxicity measurements with biosensors, part of it was extracted to hexane for determination of oxidation efficiency by GC or GC/MS.

\section{Toxicity tests}

The immobilization of $\mathrm{AChE}$ to $\mathrm{CPG}$ was carried out according to the procedure published elsewhere [16]. The CPG beads with immobilized enzyme were stored at $4{ }^{\circ} \mathrm{C}$ in phosphate buffer ( $\mathrm{pH}$ 6.0). Before use, the immobilized enzyme was packed into a PEEK column $(60 \times 2.1 \mathrm{~mm}$ i.d. $)$. To assemble the photothermal biosensor, the bioanalytical column was incorporated into an FIA manifold. The carrier buffer has been continuously pumped through the system by an HPLC pump at a $0.5 \mathrm{~mL} / \mathrm{min}$ flow rate. To determine the enzyme activity, $20 \mu \mathrm{L}$ of the substrate [acetylthiocholine iodide (ASChI)] containing 5,5'-dithiobis (2-nitrobenzoic acid) (DTNB) was injected onto the bioanalytical column to generate 5-thio-2-nitrobenzoate [19], which exhibits an absorption maximum at $410 \mathrm{~nm}$. A spectrophotometer (HP 8453) or a dual-beam (pump-probe configuration) thermal lens spectrometer similar to 
the instrument described elsewhere [13], and utilizing the excitation laser light at $476 \mathrm{~nm}(100 \mathrm{~mW})$ was used as a transducer of the bioanalytical signal.

The sample solution was injected via $1 \mathrm{~mL}$ injection loop, and the remaining enzyme activity determined as just described. The pesticide's concentration was determined for samples showing the degree of inhibition higher than $10 \%$ limit of detection (LOD), based on the standard addition method utilizing additions of $10 \mathrm{ng}$ malaoxon/1 $\mathrm{mL}$ of sample. Results were expressed as malaoxon equivalents.

\section{RESULTS AND DISCUSSION}

\section{Photochemical degradation of coumaphos and azinphos-methyl}

Coumaphos and azinphos-methyl were selected as model compounds in this study of photochemical transformations. The structure of these two insecticides differs mainly in the bridging atom between the leaving group and the central phosphorus atom. The leaving groups in both compounds are large heterocyclic rings which are bonded with oxygen to central phosphorus atom in the first case and with sulfur in the case of azinphos-methyl. The rearrangement of the structure and the formation of more toxic oxo compound in the case of coumaphos is possible.

The photodegradation of coumaphos was followed in the air, in oxygen, and in nitrogen atmosphere (Fig. 1). It was observed that the fastest reaction occurred in nitrogen atmosphere where the formation of a new compound was also detected by HPLC as a chromatographic peak at 18.5 min (Fig. 2). This was already detected in samples taken immediately after starting the irradiation process, while the maximal concentration of the compound was observed between 5 and $10 \mathrm{~min}$ of irradiation, and started to decrease afterwards. After 90 min of irradiation, the chromatographic peak at 18.5 min was not detected anymore. No formation of similar compounds or other photodegradation products was observed when coumaphos was irradiated in oxygen or in the air.

Since the observed photoproduct shows similar absorption spectrum (obtained by diode arrays) as that of the parent compound and based on the fact that its formation is much more important in the absence of oxygen, we can assume that it is most probably the result of a simple rearrangement.

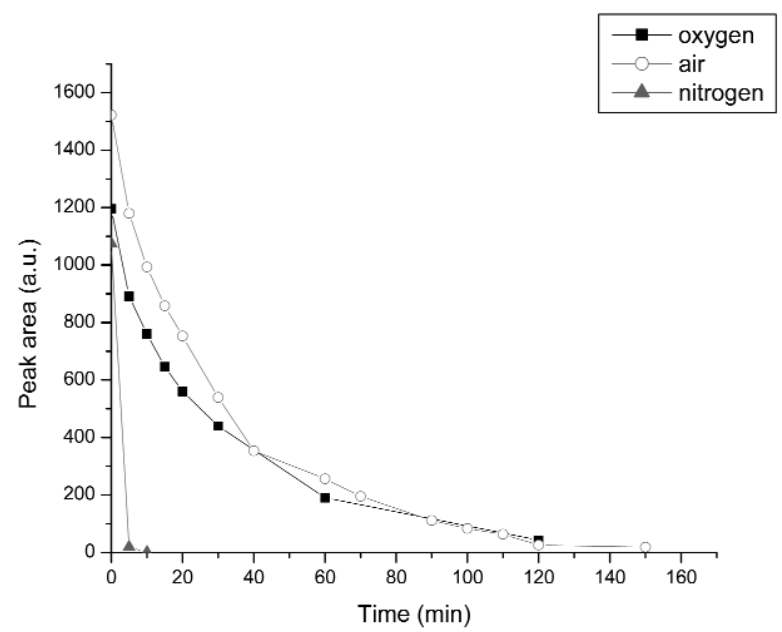

Fig. 1 Photodegradation of coumaphos (initial concentration $0.011 \times 10^{-3} \mathrm{M}$ ) in the air, in the presence of oxygen, and in the presence of nitrogen at $22{ }^{\circ} \mathrm{C}$. 


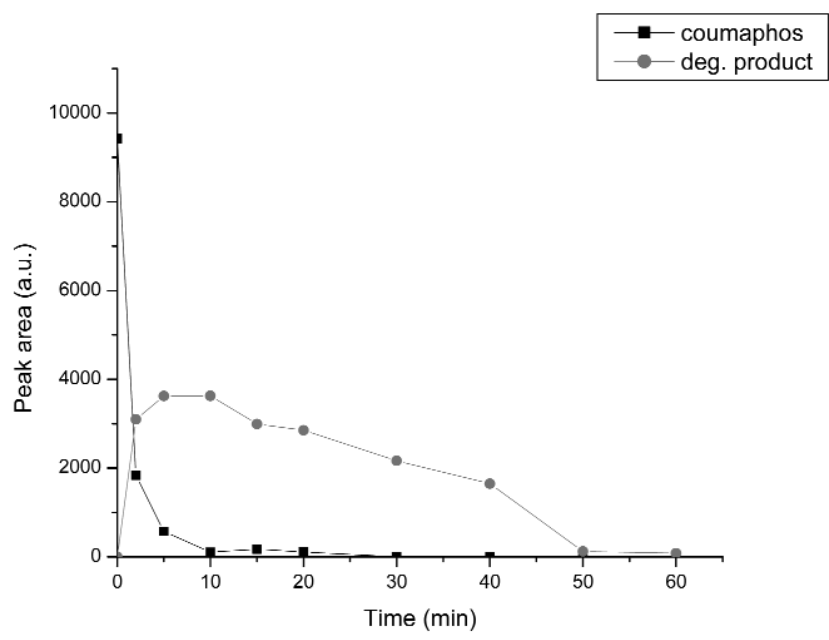

Fig. 2 Photodegradation of coumaphos (initial concentration $0.011 \times 10^{-3} \mathrm{M}$ ) and formation of rearranged product in nitrogen atmosphere at $22{ }^{\circ} \mathrm{C}$.

The proposed structural formula of this compound is given on Fig. 3 (compound X), which fully explains the similarity of the absorption spectra. Other photodegradation products obtained in the presence of oxygen were not detected most probably because of their coelution with the solvent front.

On the contrary, in the case of azinphos-methyl, no additional signals in HPLC chromatograms, which would correspond to products similar to compound X (Fig. 3), were observed.<smiles></smiles>

Fig. 3 Rearrangement of coumaphos during photodegradation in nitrogen and in the presence of oxygen.

\section{Laser flash photolysis identification of short-lived intermediates}

Laser flash photolysis (LFP) experiments were undertaken in order to elucidate the nature of the shortlived intermediate formed after irradiation of coumaphos and also to explain the effect of oxygen on the formation of product $\mathrm{X}$. 
As clearly shown in Fig. 4a, the 355-nm laser excitation leads to a transient absorption spectrum in the range 350-600 $\mathrm{nm}$. The observed transient (T) presents a spectrum with maxima at 390 and 430 $\mathrm{nm}$. There is also bleaching with a maximum at $318 \mathrm{~nm}$ corresponding to the disappearance of coumaphos in its ground state. The reactivity of the transient species $\mathrm{T}$ with oxygen was carefully examined. As observed in Fig. 4b, T efficiently reacts with oxygen. The lifetime was evaluated to 20 and $1.2 \mu \mathrm{s}$ in oxygen saturated and deaerated solutions, respectively. These results lead us to the conclusion that the transient $\mathrm{T}$ is more likely the coumaphos triplet state. The rate constant for its reaction with oxygen was evaluated to $3.1 \times 10^{9} \mathrm{~mol}^{-1} \mathrm{~L} \mathrm{~s}^{-1}$. These results clearly indicate that the formation of photoproduct $\mathrm{X}$ involves the triplet excited state of coumaphos.
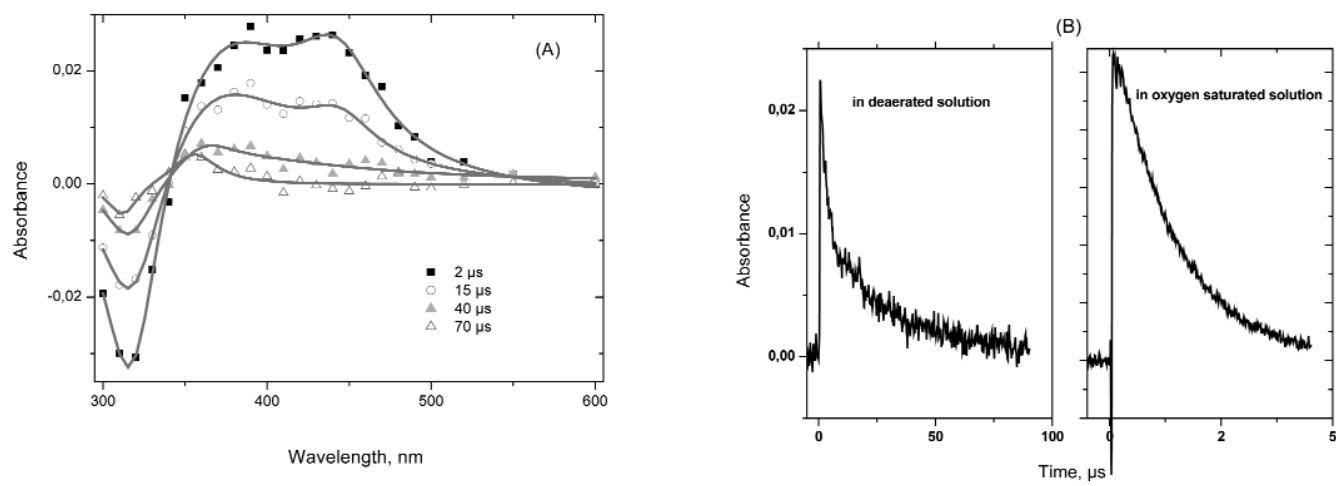

Fig. 4 (A) transient absorption spectra observed at various times after the LFP in a deaerated solution of coumaphos. (B) Traces at $400 \mathrm{~nm}$ in deaerated and oxygen-saturated solution.

\section{Enzymatic oxidation of thiono compounds to oxo derivatives}

An essential step in bioanalytical determination of OP pesticides is the oxidation of thiono compounds into oxo derivatives, which are actual inhibitors of ChEs in organisms after the oxidation of parent compounds in the body. This can be a quite time-consuming procedure, which might not yield $100 \%$ oxidation efficiency at the end. As evident from Fig. 5, only about $50 \%$ oxidation efficiency can be obtained for pesticides such as malathion, or even less for diazinon and fenitrothion after a $10 \mathrm{~min}$ oxidation with NBS following the procedure described in the experimental section. To avoid longer oxidation times, which are needed to achieve lower LOD of the assay, enzymatic oxidation methods [20] are being tested. They should enable on-line oxidation of thiono compounds in an FIA system, by using an oxidation column with an immobilized enzyme, as already indicated in Fig. 6. At present, high oxidation efficiencies (over $85 \%$ ) were obtained instantaneously for malathion in a batch mode when using chloroperoxidase as an oxidizing enzyme. 


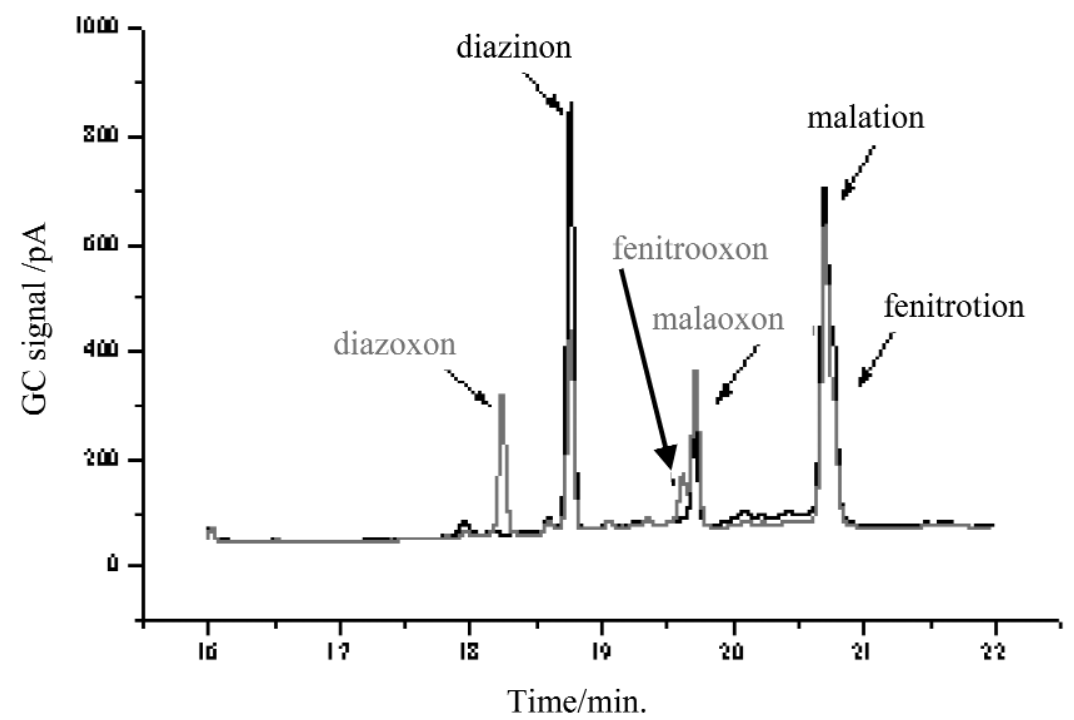

Fig. 5 Gas chromatograms of a mixture of OP insecticides obtained before (black) and after oxidation by NBS (gray). Initial concentrations of parent pesticides were as follows: $11.1 \mathrm{mg} / \mathrm{L}$ for fenitrotion, $16.6 \mathrm{mg} / \mathrm{L}$ for diazinon, and $18.9 \mathrm{mg} / \mathrm{L}$ for malation.

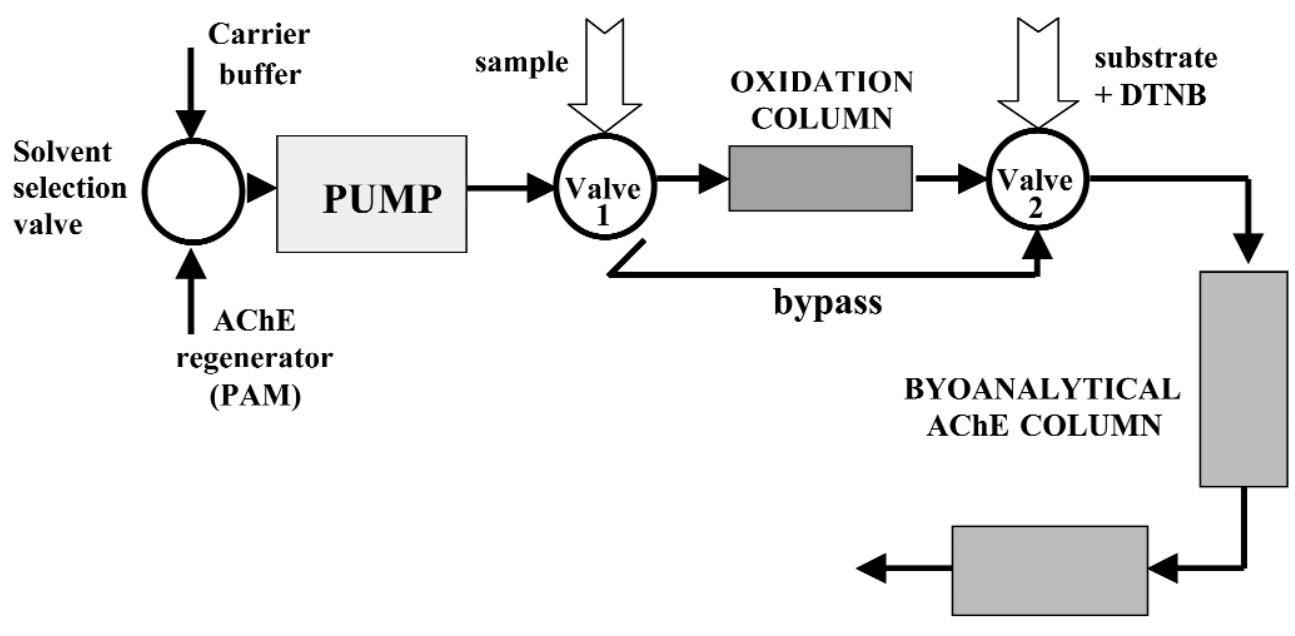

DETECTION CELL

Fig. 6 Schematic diagram of a bioanalytical FIA system for detection of OP pesticides and related AChE-inhibiting compounds.

\section{Bioanalytical FIA system for detection of OP pesticides}

One of the most crucial steps in any bioanalytical assay is the incubation time required for inhibition reaction as well as for the hydrolysis of substrate during the determination of remaining enzyme activity. In FIA systems, the incubation times are determined primarily by the flow rate of the carrier buffer. For the experimental set-up used in this work (Fig. 6) the contact time was expressed as the time required for the sample to elute through the bioanalytical column. It is evident from Fig. 7 that a contact time of about $10 \mathrm{~min}$ is required to achieve the maximum sensitivity of the assay. However, the time of the assay can again be reduced only for the cost of lower sensitivity or best possible LOD since the inhibition efficiency at faster flow rates is considerably lower. For example, for a 2-min contact time 


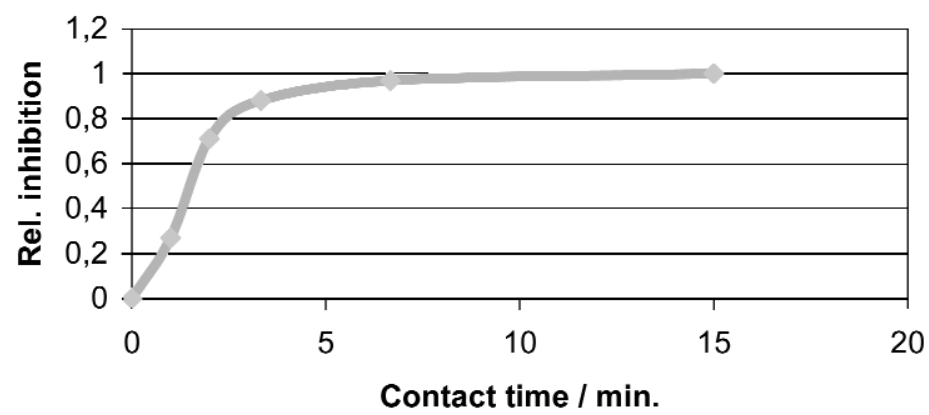

Fig. 7 Dependence of inhibition on the contact time between the sample and immobilized AChE in the bioanalytical column.

$(0.5 \mathrm{~mL} / \mathrm{min})$, about $70 \%$ inhibition efficiency is achieved, and it further drops to only about $20 \%$ for $1 \mathrm{~min}$ contact time, which is achieved at $1 \mathrm{~mL} / \mathrm{min}$ flow rate.

For detection of oxo OPs, the described FIA bioanalytical system, despite lower inhibition efficiency, still provides about 50 times lower limits of detection when compared to maximum residue levels for OP compounds in foodstuffs [21,22] and a sample throughput of about 10 samples/h (sample preparation not accounted for). This can be achieved when a $0.5 \mathrm{~mL} / \mathrm{min}$ flow rate of the carrier buffer is combined with appropriate timing in the substrate-sample-substrate injection sequence. These LOD values are accessible even with the application of spectrophotometric detection as demonstrated on Fig. 8, where two injections of substrate are followed by the injection of $200 \mu \mathrm{L}$ of a fruit juice containing $50 \mathrm{ppb}$ of malaoxon, and one injection of substrate which reflects about $50 \%$ reduced activity of the AChE. The possibility of using spectrophotometric detection is of great importance for routine applications in food production and general screening of food quality and safety.

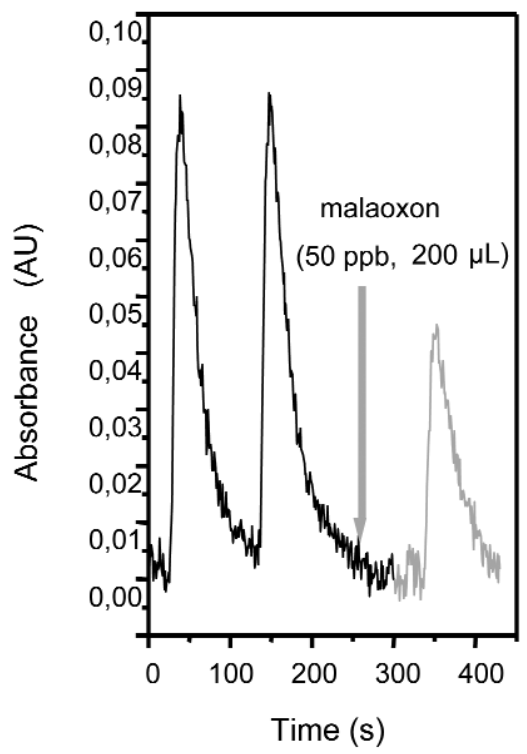

Fig. 8 Spectrophotometric detection of the bioanalytical signal showing two initial injections of the substrate (black), and the reduction of AChE activity after the injection of $200 \mu \mathrm{L}$ sample with $50 \mathrm{ppb}$ malaoxon (shown by the arrow) reflected in the third injection of substrate (gray). 
Further increase in sample throughput, which would be of great interest, for example, for control of drinking water quality and protection of general public against terrorist actions, is, however, possible only with the increase of the carrier buffer flow rate and eventually the reduction of sample volume, which consequently both require the use of a highly sensitive detection technique such as TLS. TLS can provide limits of detection up to 100 times lower compared to spectrophotometry, enabling thus the detection of OP compounds such as paraoxon at sub-ppb levels, as already shown by the applications of bioanalytical FIA assays for vegetables, fruit juices, and water [16,18].

For the purpose of testing, the described bioanalytical FIA assay was applied in practice for determination of oxo OP pesticides in 60 samples of fruit juice concentrates and blended fruits prior to their processing in fruit juice production. As confirmed by parallel determinations of OP compounds by GC and GC/MS, no false negative results were obtained. This is of high relevance, since a reliable screening test should not give clearance to a sample containing OP compounds. On the other hand, among 60 tested samples, only one sample was identified as problematic due to an observed $15 \%$ inhibition of AChE, which corresponded to $150 \mathrm{ppb}$ of malaoxon equivalents in the sample. This was still under the $500 \mathrm{ppb}$ maximal residue level (MRL) value, but since no presence of OP pesticides was detected by GC/MS, it represented a false positive result. This corresponds to a $1.7 \%$ level of false positives for the given set of test samples. False positives are, however, less problematic since a positive result of a screening test just means that the sample should be verified by standardized analytical methods.

\section{CONCLUSIONS}

The photodegradation study of coumaphos and azinphos-methyl has shown that beside the degradation of parent pesticides, some transformation products can be formed under specific conditions as well. In the case of coumaphos, the rearrangment of substituents occurred in the absence of oxygen and was confirmed by LFP with the observation of long-lived triplet excited state in nitrogen atmosphere. This was not the case for azinphos-methyl owing to different bridging atoms in the structure of two compounds. The transformation of coumaphos, which leads to the formation of a more toxic oxo organophosphate, confirms the need for toxicity testing of photodegraded samples containing OP compounds.

The suitability of bioanalytical FIA systems utilizing spectrophotometric or photothermal detection such as TLS for rapid and sensitive screening in food quality control was demonstrated. Owing to the high sensitivity of TLS, several steps in sample preparation can be avoided (preconcentration, purification, isolation) and reaction times reduced. High sample throughputs $\left(10 \mathrm{~h}^{-1}\right)$ with LODs still about 50 times below MRL are possible for detection of medium toxic OP compounds already with the use of spectrophotometric detection. Despite a relatively small set of test samples, it was observed that the method shows a low level (1.7 \%) of false positive results and, more importantly, no false negative results were obtained.

Further improvements are possible with the application of TLS detection, on-line biochemical oxidation of thiono OP compounds, increase of carrier buffer flow rate, and reduction of sample volume. These are the subject of our current investigations.

\section{ACKNOWLEDGMENTS}

This work was supported by the Ministry of Education, Science, and Sport of the Republic of Slovenia and by the Leonardo da Vinci Programme (project number SI/03/A/EX/176108). 


\section{REFERENCES}

1. W. H. Chambers. "Organophosphorus compounds: An overview", in Organophosphates, Chemistry, Fate, and Effects, pp. 3-17, Academic Press, New York (1992).

2. K. D. Racke. "Degradation of organophosphorus insecticides in environmental matrices", in Organophosphates, Chemistry, Fate, and Effects, pp. 47-73, Academic Press, New York (1992).

3. S. O. Pehkonen and Q. Zhang. Crit. Rev. Environ. Sci. Technol. 32 (1), 17-72 (2002).

4. V. H. Freed, C. T. Chiou, D. W. Schmedding. J. Agric. Food Chem. 27, 706-708 (1979).

5. J. A. Noblet, L. A. Smith, I. H. Stuffet. J. Agric. Food Chem. 44, 3685-3693 (1996).

6. A. Dannenberg and S. O. Pehkonen. J. Agric. Food Chem. 46, 325-334 (1998).

7. K. C. Dowling and A. T. Lemley. J. Environ. Sci. Health B 30 (5), 585-604 (1995).

8. R. S. Givens and L. W. Kueper. Chem. Rev. 93, 55-66 (1993).

9. G. Durand, J. L. Abad, F. Sanchez-Baeza, A. Messeguer, D. Barcelo. J. Agric. Food Chem. 42, 814-821 (1994).

10. Z. Mengyue, C. Shifu, T. Yaowu. J. Chem. Tech. Biotechnol. 64, 339-344 (1995).

11. R. Doong and W. Chang. J. Photochem. Photobiol. A: Chem. 107, 239-244 (1997).

12. R. T. Meijers, E. J. Oderwald-Muller, P. A. N. M. Nuhn, J. C. Kruithof. Ozone Sci. Eng. 17, 673-686 (1995).

13. P. Roche and M. Prados. Ozone Sci. Eng. 17, 657-672 (1995).

14. P. Skládal. Food Technol. Biotechnol. 34, 43 (1996).

15. M. F. Leon-Gonzalez and A. Townshend. J. Chromatogr. 539, 47-54 (1991).

16. L. Pogačnik and M. Franko. Biosens. Bioelectr. 14, 569-578 (1999).

17. L. Pogačnik and M. Franko. Talanta 54, 631-641 (2001).

18. L. Pogačnik and M. Franko. Biosens. Bioelectr. 18, 1-9 (2003).

19. G. L. Ellman, K. D. Courtney, V. Andres, R. M. Featherstone. Biochem. Pharmacol. 7, 88-95 (1961).

20. J. Hernandez, N. R. Robledo, L.Velasco, R. Quintero, M. A. Pickard, R. Vazquez-Duhalt. Pest. Biochem. Physiol. 61, 87-94 (1998).

21. British Crop Protection Council. The Pesticide Manual (1997).

22. Official Gazette of the Republic of Slovenia, 54, 8.7.1999. Pravilnik o mejnih vrednostih pesticidov v oziroma na živilih rastlinskega izvora. 